\title{
The ASM Refinement Method
}

\author{
Egon Börger \\ University of Pisa, Pisa, Italy
}

\begin{abstract}
In this paper the abstract state machine (ASM) refinement method is presented. Its characteristics compared to other refinement approaches in the literature are explained. Some frequently occurring forms of ASM refinements are identified and illustrated by examples from the design and verification of architectures and protocols, from the semantics and the implementation of programming languages and from requirements engineering.
\end{abstract}

Keywords: Models of computation; Specifying and Verifying and Reasoning about Programs

\section{Introduction}

Refinement is a general methodological principle which is present wherever a complex system or problem is described piecemeal, decomposing it into constituent parts which are detailed in steps to become manageable. Refinement goes together with the inverse process of abstraction which has characterised mathematics since ancient times. In software engineering stepwise refinement in one way or an other underlies all top-down approaches. Not surprisingly it also played a central role in incremental program development and verification in the structural programming endeavour (see [Wir71, Dij72, DDH72]) and since then has been studied intensively for specification and verification approaches which are based on algebraic, set-theoretic or logical methods, as is well documented in numerous books, e.g. [WoD96, Bav98, deE98, FiL98, DeB01]. Exploiting the generality of abstraction offered by the notion of abstract state machines (ASMs) [Gur85] and guided by problems of practical system design, the ASM ground model method has been developed in [Bor90a, Bor90b, Bor92, Bor94] together with a rather general refinement scheme for ASMs. Through further development in [BoR95b, BGR95, BeB96b, BeB96a, BoD96, BoM97a, BoM97b, BoS98, SSB01], ASM ground model construction and stepwise refinement became, together with the definition of $A S M$ s in [Gur95], the three constituents of the ASM method for practical system design and analysis (see the AsmBook [BoS03] for a systematic introduction and [Bor02b] for historical details). In the ASM method, stepwise refinement appears as a practical method for crossing levels of abstraction to link ASM models through well-documented incremental development steps, starting from ground models and turning them piecemeal into executable code.

In this paper we explain the ASM refinement method and characterise it with respect to other refinement approaches in the literature. We show that the ASM refinement method provides a kind of meta-framework which integrates well-known more specific notions of refinement (see [Bac79, Mor87, Mor94, Bav98, deE98, DeB01]), 
similarly to the way the notion of ASMs covers well-known models of computation and approaches to system design [Bor03]. For readers who are not familiar with ASMs we provide in Section 2 a summary of the few basic definitions which are needed for a technical understanding. In Section 3 we define the general notion of ASM refinement. In Section 4 we illustrate the ASM refinement concept using some practically useful specialisations, namely conservative extension, procedural (submachine) refinement, pure data refinement and instantiation. In Section 5 we present a general scheme for proving the correctness of refinements which can be used to establish complex system properties by proving them in an abstract model which is correctly refined to the considered system. In the Conclusion we briefly review some main usages of ASM refinements in practical system design and analysis. The focus in this survey paper is on an illustration of principles and usage of ASM refinements, capturing various forms of refinement within one conceptual framework, rather than on a formalised technical development. For the illustrations we use a variety of examples from different application domains to document the wide range spanned by the refinement approach.

\section{The Notion of Abstract State Machines}

In this section we recall the basic definitions concerning ASMs and illustrate them using a simple example. For a more detailed definition of these terms we refer the interested reader to Section 2.4 of the AsmBook [BoS03].

\subsection{Basic ASMs and their Runs}

An ASM is a finite set of so-called transition rules of form

\section{if Condition then Updates}

which transform abstract states. Two more forms are explained below. The Condition (also called guard) under which a rule is applied is an arbitrary predicate logic formula without free variables, whose interpretation evaluates to true or false. Updates is a finite set of assignments of form

$$
f\left(t_{1}, \ldots, t_{n}\right):=t
$$

whose execution is to be understood as changing (or defining, if there was none) in parallel the value of the occurring functions $f$ at the indicated arguments to the indicated value. More precisely, in the given state first all parameters $t_{i}, t$ are evaluated to their values, say $v_{i}, v$, then the value of $f\left(v_{1}, \ldots, v_{n}\right)$ is updated to $v$ which represents the value of $f\left(v_{1}, \ldots, v_{n}\right)$ in the next state. Such pairs of a function name $f$, which is fixed by the signature, and an optional argument $\left(v_{1}, \ldots, v_{n}\right)$, which is formed by a list of dynamic parameter values $v_{i}$ of whatever type, are called locations. Location-value pairs $(l o c, v)$ are called updates.

The notion of ASM states is the classical notion of mathematical structures where data come as abstract objects, i.e., as elements of sets (also called domains or universes, one for each category of data) which are equipped with basic operations (partial functions in the mathematical sense) and predicates (attributes or relations). For the evaluation of terms and formulae in an ASM state, the standard interpretation of function symbols by the corresponding functions in that state is used. Without loss of generality we usually treat predicates as characteristic functions and constants as 0 -ary functions. Partial functions are turned into total functions by interpreting $f(x)=$ undef with a fixed special value undef as $f(x)$ being undefined.

The notion of ASM run is an instance of the classical notion of computation of transition systems. An ASM computation step in a given state consists in executing simultaneously all updates of all transition rules whose guard is true in the state, if these updates are consistent, in which case the result of their execution yields the next state. In the case of inconsistency the computation does not yield a next state, a situation which typically is reported by executing engines with an error message. A set of updates is called consistent if it contains no pair of updates with the same location, i.e. no two elements $(l o c, v),\left(l o c, v^{\prime}\right)$ with $v \neq v^{\prime}$. An ASM step is performed as an atomic action with no side effects.

Simultaneous execution provides means to locally describe a global state change, namely as obtained in one step through executing a set of updates. The only limitation - imposed by the need for uniquely identifying objects residing in locations - is the consistency of the set of updates which have to be executed. The local description of global state changes also implies that by definition the next state differs from the previous state only at locations which appear in the update set. Simultaneous execution also provides a convenient way to abstract from sequentiality where it is irrelevant for the investigation. This synchronous parallelism in the ASM execution model is 
enhanced by the following notation to express the simultaneous execution of a rule $R$ for each $x$ satisfying a given condition $\varphi$ (where typically $x$ will have some free occurrences in $R$ which are bound by the quantifier):

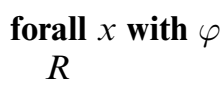

Similarly, non-determinism as a convenient way to abstract from details of scheduling of rule executions can be expressed by rules of the form

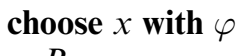

where $\varphi$ is a Boolean valued expression and $R$ a rule. The meaning of such an ASM rule is to execute rule $R$ with an arbitrary $x$ chosen among those satisfying the selection property $\varphi$. If there exists no such $x$, nothing is done.

Common notations like where, let, if-then-else are used without further explanation since they are easily reducible to the above basic definitions. Sometimes we also use the table-like case notation with pattern matching, in which case we try out the cases in the order of writing, from top to bottom. We also use rule schemes, namely rules with variables, and named parameterised rules, mainly as an abbreviational device to enhance the readability or as a macro allowing us to reuse machines and to display a global machine structure. For example

$$
\text { if } \ldots a=(x, y) \ldots \text { then } \ldots x \ldots y \ldots
$$

abbreviates

$$
\text { if } \ldots \text { ispair }(a) \ldots \text { then } \ldots f \operatorname{st}(a) \ldots \operatorname{snd}(a) \ldots
$$

sparing us the need to write explicitly the recognisers and the selectors. Similarly, an occurrence of

$$
r\left(x_{1}, \ldots, x_{n}\right)
$$

where a rule is expected stands for the corresponding rule $R$ (which is supposed to be defined somewhere else, with $r\left(x_{1}, \ldots, x_{n}\right)=R$ appearing in the declaration part of the ASM where $r\left(x_{1}, \ldots, x_{n}\right)$ is used). When such a 'rule call' $r\left(x_{1}, \ldots, x_{n}\right)$ is used, the parameters have to be instantiated by legal values (objects, functions, rules, whatever) so that the resulting rule has a well-defined semantical meaning on the basis of the explanations given above. A precise semantical definition of such ASM submachine calls has been defined in [BoS00a].

For purposes of separation of concerns it is often convenient to impose for a given ASM additional constraints on its runs to circumscribe those one wants to consider as legal. Logically speaking this means restricting the class of models satisfying the given specification. Such restrictions are particularly useful if the constraints express reasoning assumptions for a high-level machine which are easily shown to hold in a refined target machine. In general, ASMs are reactive systems which iterate their computation step, but for the special case of terminating runs one can choose among various natural termination criteria to constrain runs, namely that no rule is applicable any more or that the machine yields an empty update set or that the state does not change any more.

\subsection{Classification of Locations and Functions}

In an ASM, a priori no restriction is imposed neither on the abstraction level nor on the complexity nor on the means of definition of the functions used to compute the arguments and the new value denoted by $t_{i}, t$ in function updates. In support of the principles of separation of concerns, information hiding, data abstraction, modularisation and stepwise refinement, the ASM method exploits, however, the following distinctions reflecting the different roles these functions (and more generally locations) can assume in a given machine.

The major distinction for a given ASM $M$ is between its static functions - which never change during any run of $M$ so that their values for given arguments do not depend on states of $M$ - and dynamic ones which may change as a consequence of updates by $M$ or by the environment (read: by some other - say an unknown - agent representing the context in which $M$ computes), so that their values for given arguments may depend on states of $M$. By definition static functions can be thought of as given by the initial state, so that where appropriate, handling them can be clearly separated from the description of the system dynamics. Whether the meaning of these functions is determined by a mere signature ('interface') description, or by axiomatic constraints, or by an abstract specification, or by an explicit or recursive definition, or by a program module, depends on the degree of information hiding the specifier wants to realise. Static 0 -ary functions represent constants, whereas with dynamic 
0 -ary functions one can model variables of programming (not to be confused with logical variables). Dynamic functions can be thought of as a generalisation of array variables or hash tables.

The dynamic functions are further divided into four subclasses. Controlled functions (for $M$ ) are dynamic functions which are directly updatable by and only by the rules of $M$, i.e., functions $f$ which appear in at least one rule of $M$ as leftmost function (namely in an update $f(s):=t$ for some $s, t$ ) and are not updatable by the environment (or more generally by another agent in the case of a multi-agent machine). These functions are the ones which constitute the internally controlled part of the dynamic state of $M$.

Monitored functions, also called in functions, are dynamic functions which are read but not updated by $M$ and directly updatable only by the environment (or more generally by other agents). They appear in updates of $M$, but not as leftmost function of an update. These monitored functions constitute the externally controlled part of the dynamic state of $M$. To describe combinations of internal and external control of functions, one can use interaction functions, also called shared functions, defined as dynamic functions which are directly updatable by rules of $M$ and by the environment and can be read by both (so that typically a protocol is needed to guarantee consistency of updates). The concepts of monitored and shared functions allow one to separate in a specification computation from communication concerns. In fact the definition does not commit to any particular mechanism (e.g. message passing via channels) to describe the exchange of information between an agent and its environment (and similarly between arbitrary agents in the case of a multi-agent machine). As with static functions the specification of monitored functions is open to any appropriate method. The only assumption made is that in a given state the values of all monitored functions are determined.

Out functions are dynamic functions which are updated but not read by $M$ and are monitored (read but not updated) by the environment or in general by other agents. Formally, such output functions do appear in some rules of $M$, but only as the leftmost function of an assignment.

Functions are called external for $M$ if for $M$ they are either static or monitored.

An orthogonal, pragmatically important classification comes through the distinction of basic and of derived functions. Basic functions are functions which are taken for granted (declared as 'given', typically those forming the basic signature); derived functions are functions which even if dynamic are not updatable either by $M$ or by the environment but may be read by both and yield values which are defined by a fixed scheme in terms of other (static or dynamic) functions (and as a consequence may sometimes not be counted as part of the basic signature). Thus derived functions are sort of auxiliary functions coming with a specification or computation mechanism which is given separately from the main machine; they may be thought of as a global method with read-only variables.

The same classification principle is applied to (sets of) locations or updates.

\subsection{Multi-Agent ASMs}

A multi-agent $A S M$ is defined as a set of agents each of which executes its own basic ASM. This may happen in a synchronous or in an asynchronous manner. In a synchronous ASM the agents execute their basic ASM in parallel, synchronised using an implicit global system clock. Semantically a synchronous ASM is equivalent to the set of all its constituent single-agent ASMs, operating in the global states over the union of the signatures of each component, though each agent is equipped with its own set of states and rules. This concept allows one to define and analyse the interaction between components using precise interfaces over common locations.

A problem one has to solve for runs of asynchronously cooperating agents originates in the possible incomparability of their moves which may come with different data, clocks, moments and duration of execution, making it difficult to uniquely define a global state where moves are executed to locate changes of monitored functions in an ordering of moves. A coherence condition in the definition of asynchronous multi-agent ASM runs given in [Gur95] postulates well-definedness for a relevant portion of state in which an agent is supposed to perform a step, thus providing a notion of 'local' stable view of 'the' state in which an agent makes a move. The underlying synchronisation scheme is described using partial orders for moves of different agents which reflect causal dependencies, determining which move depends upon (and thus must come 'before') which other move. This synchronisation scheme is as liberal as it can be, restricted only by the consistency condition for the updates which is logically indispensable, and thus can be instantiated by any consistent synchronisation mechanism.

Formally a run of an asynchronous ASM, also called partial order run, is defined as a partially ordered set $(M,<)$ of moves $m$ (read: rule applications) of its agents satisfying the following conditions: 
- finite history: each move has only finitely many predecessors, i.e. for each $m \in M$ the set $\left\{m^{\prime} \mid m^{\prime}<m\right\}$ is finite;

- sequentiality of agents: the set of moves $\{m \mid m \in M, a$ performs $m\}$ of every agent $a \in$ Agent is linearly ordered by $<$;

- coherence: each finite initial segment (downward closed subset) $X$ of $(M,<)$ has an associated state $\sigma(X)-$ think of it as the result of all moves in $X$ with $m$ executed before $m^{\prime}$ if $m<m^{\prime}$ - which for every maximal element $m \in X$ is the result of applying move $m$ in state $\sigma(X-\{m\})$.

The coherence condition immediately implies, for every finite initial segment $X$ of a run of an asynchronous ASM, that all linearisations of $X$ yield runs with the same final state. The definition provides no clue to construct partial order runs for an asynchronous ASM, but it makes the freedom explicit one has in implementing the described causal dependencies of certain local actions of otherwise independent agents. Notably the definition imposes no fairness condition on runs.

\subsection{Example Machine: Java Interpreter}

We illustrate the above definitions by an elementary but real-life machine used for defining the semantics of Java, chosen among the submachines that constitute an ASM interpreter for Java programs. We take care to exemplify how a formalisation that is guided by the language features in question leads to a simple ASM ground model. Below we will use these ground model rules to illustrate a typical ASM refinement of this interpreter to one for (e.g. Java) programs that have been compiled to Java Virtual Machine (JVM) bytecode. The material is extracted from [SSB01], where the reader can find the complete machines which we cannot reproduce here.

The two ASM interpreters for Java programs and for JVM bytecode respectively are factored into five parallel submachines, isolating language features which represent milestones in the evolution of modern programming languages and of the techniques for their compilation, namely imperative (sequential control), procedural (module or static class), object-oriented, exception handling, and concurrency (thread) features. This decomposition of the interpreters into a sequence of conservative extensions (see the definition below) supports not only design modularity, but also the splitting of the correctness proof into a series of lemmas. It is formally reflected by the fact that the single-thread Java interpreter EXECJAVA is defined as a composition of the following submachines, one for each language layer:

$$
\begin{aligned}
& \text { EXECJAVA }= \\
& \text { EXECJAVA }_{I} \\
& \text { EXECJAVA }_{C} \\
& \text { EXECJAVA }_{O} \\
& \text { EXECJAVA }_{E} \\
& \text { EXECJAVA }_{T}
\end{aligned}
$$

Depending on the scheduling policy, multiple-thread Java programs can be interpreted by enriching EXECJAVA with an appropriate scheduler ASM, which at each moment schedules one thread for execution (as done in [SSB01]), or by an asynchronous ASM in the sense defined above, where each thread is interpreted by an agent executing the instantiation of EXECJAVA for this thread.

Reflecting the two major syntactical constituents of Java programs, namely expressions and statements, each submachine is decomposed into a submachine for expression evaluation and a submachine for the execution of statements of that language layer. For example

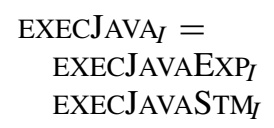

View program execution as a walk through the annotated abstract syntax tree: at each position, which can be denoted by a controlled function pos representing an abstract program counter and pointing to the current expression or statement to be elaborated or to the result of its elaboration, the corresponding phrase is evaluated or executed and then the control flow proceeds to the next phrase (at the same or the next position). Positions are displayed as superscripts, for example, as in ${ }^{\alpha} \exp$ or in ${ }^{\alpha} \mathrm{stm}$.

A controlled function restbody: Pos $\rightarrow$ Phrase, which initially contains the to-be-interpreted input program body, is used to record (a) the still-to-be-executed part of the current program (method body), (b) the already computed and still needed expression values, and (c) information about the current abruption or the successful termination of the computation. More precisely, restbody(pos) denotes the currently to-be-computed subterm 
of restbody at pos: it will eventually be replaced by the computed value of the subexpression, or by a reason for abruption, ${ }^{1}$ or by the constant Norm, which denotes successful termination (also called normal completion) of a statement.

The current value of the local variables is kept in a local environment function locals, a controlled function that associates local variables with their current values; the latter are updated upon execution of assignment statements or of an expression evaluation (in the latter case one commonly speaks of the update as a side effect, though it is an integral part of the dynamic state change).

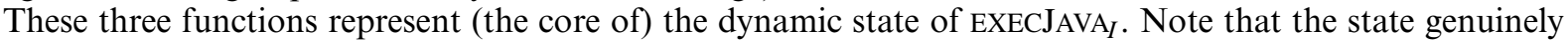
consists of abstract functions, not only of variables as encountered in many other state-based approaches to system design. The transition rules below describe how restbody, pos, locals, change over time by evaluating expressions and executing statements of the input program. Initially restbody is the given method body, pos is its start position firstPos and locals is empty. When the machine encounters an stm to be executed or an exp to be evaluated, or when it comes (back) to the firstPos of its method body, the context of the pending subcomputation of this phrase is given by restbody(pos). After this subcomputation has been completed, either normally or abruptly, the control passes to the parent position where the decision is taken on how to continue, either by computing the next subphrase of the phrase in the parent position, or by executing on the computed subphrases the computation to be done at the parent position. In both cases the context of the completed subcomputation changes to the superphrase restbody (up(pos)), where the function up yields the parent position of a position. Like all the other auxiliary functions, $u p$ belongs to the state of the interpreter and is static if the program is considered as loaded initially. It allows one to retrieve for a phrase, occurring in a position, the next enclosing phrase.

This context switch can be captured by the following definition, where Bstm denotes the set of block statements and Exp that of expressions:

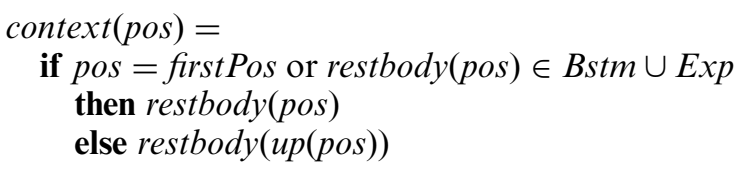

Since the context switch is often accompanied by passing to the parent position the result of the completed subcomputations, or what has to be computed from them at the parent position, we use the following rules, where $\exp [$ res $/ p]$ denotes the result of replacing the subexpression of exp at position $p$ by res. The first rule combines passing a result, from a substructure at pos to its direct superstructure, with an update of pos to the position of the superstructure. The second rule is used when a result has to be recorded without changing the value of pos.

$$
\begin{aligned}
& \text { yield } U p(\text { result })= \\
& \quad \text { restbody }:=\text { restbody }[\text { result } / \text { up }(\text { pos })] \\
& \text { pos }:=\text { up }(\text { pos }) \\
& \text { yield }(\text { result })=\text { restbody }:=\operatorname{restbody}[\text { result } / \text { pos }]
\end{aligned}
$$

We abbreviate that the machine is positioned on a direct subphrase $t$ of a structure $f(\ldots t \ldots)$ by writing $s=f(\ldots \triangleright t \ldots)$ for $s=f(\ldots t \ldots) \wedge \operatorname{pos}=\triangleright$. Similarly we write $\left.s=\operatorname{phrase}^{\triangleright} t\right)$ for $s=\operatorname{phrase}(t) \wedge$ pos $=\triangleright \wedge$ restbody $($ pos $)=t$.

This explains the definition of the machine EXECJAVAEXPI as consisting of the rules below. They reflect that all expressions, which are built up from literals and local variables by unary and binary operators, are evaluated from innermost to outermost. ${ }^{2}$ For this purpose the current control is transferred, by updating pos, from unevaluated expressions to the appropriate subexpressions, until an atom (a literal or a local variable) is reached. For binary expressions also the left-to-right evaluation strategy is taken into account, as required in Sun's manual, and for conditional expressions the condition is evaluated first. When the current task restbody (pos) requires to evaluate a context which is an atom or a compound expression all of whose relevant arguments are evaluated, this context is replaced by the (to be) computed value - which is determined for literals and for compound expressions by the static external JLS function, which is defined in Sun's Java language specification, and for variables by the local environment function locals.

\footnotetext{
1 In EXECJAVA $I$ abruptions can happen due to the restricted jump statements 'break Lab; ' and 'continue Lab; '. In EXECJAVA $C$ and EXECJAVA $E$ this is refined by return and exception values which may occur as result of return statements or exceptions.

2 One can show that this evaluation order is one among various possible refinements of a more abstract expression evaluation model. These refinements characterise the evaluation mechanisms adopted in some mainstream current programming languages, see [ZiD03].
} 


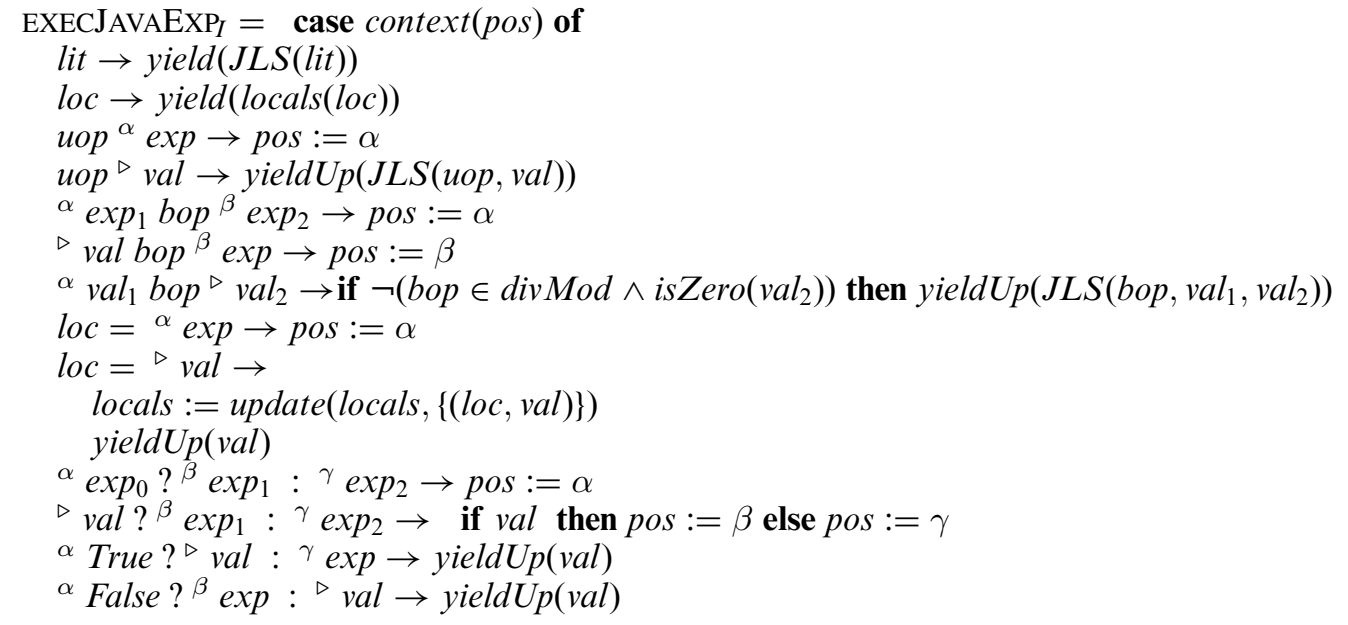

\section{Definition of ASM Refinements}

In this section we define the notion of ASM refinement and its frequently used and practically important specialisations to conservative extension, procedural (submachine) refinement, pure data refinement and instantiation.

Most established refinement notions are based upon some a priori principle. An example is the substitutivity principle, which is usually expressed as follows, quoted from [DeB01, p. 47]:

Principle of substitutivity: it is acceptable to replace one program by another, provided it is impossible for a user of the programs to observe that the substitution has taken place.

Refinement notions which are tailored to match such an a priori epistemological principle as a result may be restricted in various ways, limiting their range of applicability. Among the restrictions of this sort are the following ones one can find in one form or another in [Bac79, WoD96, Abr96, deE98, Bav98, FiL98, DeB01]:

- Restriction to certain forms of programs, often viewed as sequences of operations (straight-line programs). As a consequence, programs which are refined in this way are even structurally equivalent to their abstract counterpart, i.e. with corresponding operations occurring in the same places, thus precluding to analyse the role of other forms of control for refinement, e.g. various forms of parallelism or iteration (as encountered, for example, in some process algebraic refinement notions).

- Restriction to programs with only monolithic state operations, expressed by global functions on the state without the possibility to modify elements of the state. This makes it difficult to exploit combinations of local effects for overall refinements and leads to the well-known frame problem, which typically makes formal specifications of programs more difficult to write and to read than the programs that they describe.

- Restriction to observations interpreted as pairs of input/output sequences or of pre-post states, often with the same input/output representation at the abstract and the refined level. Such a focus on functional input/output behaviour of terminating runs or on pre-post states of data-refined operations precludes to relate arbitrary segments of abstract and refined computations. As a consequence, to be compared invariants of abstract and refined programs are viewed in terms of pre- or post-condition strengthenings or weakenings, which restricts a more general analysis of the effect of invariants as retrenchment of the class of possible models. The fact that often no change of input/output representation is permitted also precludes the possibility to refine 'abstract input', e.g. input coming in the form of monitored data, by 'controlled data' which are computed through concrete computation steps.

- Restriction to logic or proof-rule oriented refinement schemes. Tailoring refinement schemes to fit a priori fixed proof principles quickly leads to severe restrictions of the design space.

In contrast, the ASM refinement method is not based upon any concrete principle, but is rooted in mathematics (which includes formalised logic as a tiny fraction). One can make up the notion of refinement one needs, adapting to what the investigated system levels (read: the chosen levels of abstraction) demand for comparing program runs. This openness does not imply that the designer is left with an insecure footing, since the confidence 


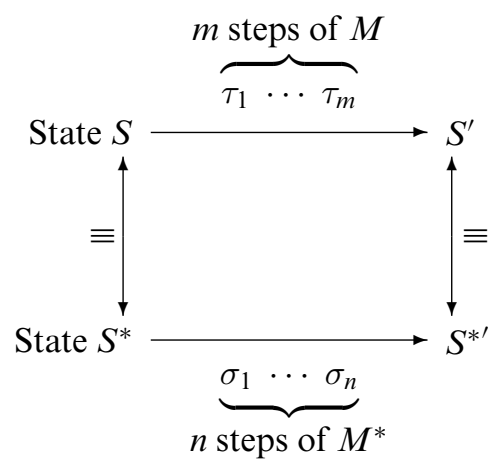

$\equiv$ is an equivalence notion between data

in locations of interest in corresponding states.

Fig. 1. The ASM refinement scheme.

given to refinements of ASM specifications to implementations goes together with the degree of mathematical precision with which the refinement is provided. The range of mathematical rigour is far more comprehensive than that of any formalised theory of logic.

The problem orientation of the ASM refinement method has shaped its development during the last decade which was driven by practical refinement tasks and geared to support divide-and-conquer techniques for both design and verification, without privileging one to the detriment of the other. What for short we call 'freedom of abstraction' offered by ASMs, i.e. the availability in ASMs of arbitrary structures to reflect the underlying notion of state, provides the necessary instrument to fine tune the mapping of a given (the 'abstract') machine to a more concrete (the 'refined') one, with its observable (typically more detailed) state and its observable (typically more involved) computation, in such a way that the intended 'equivalence' between corresponding run segments of the two ASMs becomes observable, whereby we mean that it can be explicitly defined and proved to hold under precisely stated boundary conditions.

The focus is not on generic notions of refinements which can be proved to work in every context and to provide only effects which can never be detected by any user of the new program. Instead the concern is to support a disciplined use of refinements which correctly reflect and explicitly document an intended design decision, adding more details to a more abstract design description, e.g. for making an abstract program executable, for improving a program by additional features or by restricting it through precise boundary conditions which exclude certain undesired behaviours. Exploiting the freedom of abstraction offered by ASMs one has the possibility not to let oneself get bound by an a priori commitment to particular notions of state, program, run, equivalence, or to any particular method to establish the correctness of the refinement step. The major and usually difficult task is first to listen to the subject, to find the right granularity and to formulate an appropriate refinement - or abstraction in case of a re-engineering project - that faithfully reflects the underlying design decision or re-engineering idea, and only then to look for appropriate means to justify that under the precisely stated conditions the refinement correctly implements the given model, and that the re-engineered abstract model correctly abstracts from the given code. With the ASM refinement method, whatever feasible accurate method is out there can - indeed should - be adopted, whether for verification (by reasoning) or for validation (e.g. testing model-based runtime assertions through a simulation), to establish that the intended design assumptions hold in the implementation and that refined runs correctly translate the effect of abstract ones. In particular, by appropriate instantiations of the widely open ASM refinement concept one can capture the various more restricted refinement notions studied in the literature. In this sense the ASM refinement method provides a uniform framework to reflect established specific refinement notions.

These principles can be realised based upon the general scheme for an ASM refinement step which is illustrated by the familiar commutative diagram in Fig. 1 and underlies the definition of ASM refinement below. The scheme can also be viewed as describing an abstraction step if it is used to model an implementation, as happens in re-engineering projects; see [BBG00] for an illustration using an industrial case study. 
Refinement based upon this scheme generalises in particular the standard notion of a simulation which is used to verify data refinements (see [deE98]). But it does more: to refine an ASM $M$ to an ASM $M^{*}$, as a designer one has the freedom (and the task) to define the following items:

- a notion of refined state;

- a notion of states of interest and of correspondence between $M$-states $S$ and $M^{*}$-states $S^{*}$ of interest, i.e. the pairs of states in the runs one wants to relate through the refinement, including usually the correspondence of initial and (if there are any) of final states;

- a notion of abstract computation segments $\tau_{1}, \ldots, \tau_{m}$, where each $\tau_{i}$ represents a single $M$-step, and of corresponding refined computation segments $\sigma_{1}, \ldots, \sigma_{n}$, of single $M^{*}$-steps $\sigma_{j}$, which in given runs lead from corresponding states of interest to (usually the next) corresponding states of interest (the resulting diagrams are called $(m, n)$-diagrams and the refinements $(m, n)$-refinements);

- a notion of locations of interest and of corresponding locations, i.e. pairs of (possibly sets of) locations one wants to relate in corresponding states, where locations represent abstract containers for data;

- a notion of equivalence $\equiv$ of the data in the locations of interest; these local data equivalences usually accumulate to a notion of equivalence of corresponding states of interest.

The scheme shows that an ASM refinement allows one to combine in a natural way a change of the signature (through the definition of states and of their correspondence, of corresponding locations and of the equivalence of data) with a change of the control (defining the 'flow of operations' appearing in the corresponding computation segments). Many notions of refinement in the literature keep these two features separated on purpose; see for example the notions of data refinement in VDM [FiL98] and of operation refinement in B [Abr96]. Once the notions of corresponding states and of their equivalence have been determined, one can define that $M^{*}$ is a correct refinement of $M$ if and only if every (infinite) refined run simulates an (infinite) abstract run with equivalent corresponding states, as is made precise by the following definition. By this definition, refinement correctness implies for the special case of terminating runs the inclusion of the input/output behaviour of the abstract and the refined machine, a special feature on which numerous refinement notions in the literature are focused.

Definition. Fix any notions $\equiv$ of equivalence of states and of initial and final states. An ASM $M^{*}$ is called a correct refinement of an ASM $M$ if and only if for each $M^{*}$-run $S_{0}^{*}, S_{1}^{*}, \ldots$ there is an $M$-run $S_{0}, S_{1}, \ldots$ and sequences $i_{0}<i_{1}<\ldots, j_{0}<j_{1}<\ldots$ such that $i_{0}=j_{0}=0$ and $S_{i_{k}} \equiv S_{j_{k}}^{*}$ for each $k$ and either

- both runs terminate and their final states are the last pair of equivalent states; or

- both runs and both sequences $i_{0}<i_{1}<\ldots, j_{0}<j_{1}<\ldots$ are infinite.

Often the $M^{*}$-run $S_{0}^{*}, S_{1}^{*}, \ldots$ is said to simulate the $M$-run $S_{0}, S_{1}, \ldots$ The states $S_{i_{k}}, S_{j_{k}}^{*}$ are the corresponding states of interest. They represent the end points of the corresponding computation segments (those of interest) in Fig. 1, for which the equivalence is defined in terms of a relation between their corresponding locations (those of interest). Sometimes it is convenient to assume that terminating runs are extended to infinite sequences which become constant at the final state. We refer to Fig. 1 when using the term $(m, n)$-refinement.

$M^{*}$ is called a complete refinement of $M$ if and only if $M$ is a correct refinement of $M^{*}$.

It is easy to show that in this refinement definition the sequences of corresponding states can be chosen to be minimal in the sense that between two sequence elements there are no other equivalent states, i.e. there are no $i_{k}<i<i_{k+1}, j_{k}<j<j_{k+1}$ such that $S_{i} \equiv S_{j}^{*}$.

The pairs of the initial and possibly of the final states by definition are pairs of corresponding states. Therefore refinement correctness and completeness imply for terminating runs the equivalence of any input/output behaviour of the abstract and the refined machine that is defined in terms of initial and final states and of the refinement.

In the literature correct and complete refinements with respect to terminating runs considering only the input/output behaviour appear under the name of bisimulation. Correct refinements with respect to terminating runs are known under the name of preservation of partial correctness, whereas preservation of total correctness is used for refinements adding to the correctness condition for terminating runs that every infinite refined run admits an infinite abstract run with an equivalent initial state. For details see [Sch01].

The ASM literature surveyed in [Bor02b] is full of examples of the above definition. We illustrate it here by explaining the refinement of the ASM model EXECJAVA interpreting Java programs (see Section 2) to an ASM model for an interpreter of their compiled bytecode on the Java Virtual Machine. The crux is to define how to compare runs of Java programs to equivalent runs of their compilation to bytecode on the JVM, in terms of 
exec Java and of the ASM $J V M_{E}$ defined in [SSB01, Chapters 9-13] for the stack-based JVM. The correspondence of states is defined inductively by an order-preserving function $\sigma$ which assigns to the $n$th state of a Java program run an equivalent state $\sigma(n)$ of the run of the compiled program on the JVM. In defining the equivalence notion one has to identify the relevant locations in the states of the two machines, whose value evolution guarantees that the two runs yield the same result. The definition is the result not of an a priori given refinement scheme, but of a detailed analysis of how Java instructions are interpreted, how they are compiled and how the compiled code is interpreted, covering in particular an analysis of how Java values are data refined by JVM values.

In particular, the following locations are required to have the same value in corresponding states: the current method, the class state, the global variables and the two heaps. The current positions in the programs must belong to the same phrase (read: Boolean or non-Boolean or instance creation expression, statement, value, normal or abrupt termination) and the two machines must be in the same execution phase for that phrase (entering or exiting). The values of intermediate results must be the same via some data refinement function which maps Java values, which are encoded in the function restbody, to JVM operand stack values. The local variables and the corresponding registers must contain the same values. The return addresses from possibly nested subroutines must correspond to each other. The correctness of subroutine return addresses is the most delicate part in defining what it means that in corresponding runs of execJava and of $J V M_{E}$, the Java method call frames are equivalent to the JVM stack.

To show the equivalence of corresponding states means to verify that the two machines when started on corresponding programs $P$ and compile $(P)$, upon navigating through their code, produce in corresponding method code segments the same values for (local, global, heap) variables and the same results of intermediate calculations, for the current method as well as for the still to be completed method calls. The entire section 14.1 in [SSB01] is devoted to turning the refinement correctness statement into an accurate mathematical form, just to make a correctness proof possible. We mention here only two simple cases which can be explained in terms of the expression evaluation rules of the above defined machine EXECJAVAEXP $I$ and their counterpart for the compiled code in the JVM. The correspondence for the rules evaluating literals and local variables is of type $(1,1)$, given that literals lit are compiled into JVM instructions Prim(lit) and local variables loc into JVM Load $(\overline{l o c})$ statements, where an external function is used to associate registers $\overline{l o c}$ with variables $l o c$. For the evaluation of assignment expressions two other diagram types occur due to the compilation of loc $=\exp$ by compile(exp) Dupx Store $(\overline{l o c})$, namely (a) of type $(1,0)$ for the step where EXECJAVAEXPI makes one step to update pos to the root of the exp-subtree, whereas the JVM makes no step, and (b) of type $(1,2)$ where EXECJAVAEXP I makes one step to pass the computed exp value to the parent (an assignment expression) node whereas the JVM has to make two steps, namely duplicating the value of top of the stack before storing it.

The correctness proof itself has to investigate the 83 different cases which may occur when executing Java instructions [SSB01, pp. 178-203]. It uses $(1, n)$-refinements with $0 \leqslant n \leqslant 3$ depending on the length of the computation which leads the JVM machine from one to its next state of interest (i.e. a state which corresponds in a meaningful way to a state of the Java machine). No a priori scheme was helpful in finding the right refinement relation or in establishing the correctness proof; instead we had to understand, model and investigate the behaviour of Java and of bytecode - which the freedom of abstraction in ASMs allowed us to express directly in terms of appropriate machines, avoiding any formal overhead.

\section{Frequent Refinement Types}

We present and illustrate here some specialisations to refinement patterns which turned out to be useful for practical system design and analysis. For a detailed technical comparison of various specialisations of the above ASM refinement scheme to outstanding refinement notions in the literature we refer the reader to [Sch01].

It is important for the practicability of ASM refinements that the size of $m$ and $n$ in $(m, n)$-refinements is allowed to dynamically depend on the state. An early example where $n$ has no a priori bound and can be determined only dynamically appeared in a refinement step of Prolog to WAM code in [BoR91, BoR92b, BoR95b] (see below). The ASMs for Lamport's mutual exclusion algorithm Bakery in [BGR95] provide a case where $n$ is fixed, but grows with the number of protocol members, or where $n$ is finite but without a priori bound, depending on the execution time of the participating processes. In [BoS00b] the correctness proof for exception handling in Java/JVM uses $(m, n)$-refinements, where $m$ is determined by the number of Java statements jumped over during the search for the exception handler. Although by a theorem of Schellhorn [Sch01, Th. 12] every $(m, n)$-refinement with $n>1$ can be reduced to $(m, 1)$-refinements, this is typically at the price of having more involved equivalence 
notions which may complicate the proofs. Practical experience shows that $(m, n)$-refinements with $n>1$ and including $(m, 0),(0, n)$-steps support the feasibility of decomposing complex (global) actions into simpler (locally describable) ones whose behaviour can be verified in practice. They also provide a handle to classify refinements. A comprehensive classification and study of the interesting forms of ASM refinement and of ways to compose them is still waiting to be undertaken. We list in the following some patterns which turned out to be useful for practical applications.

\subsection{Conservative Extension}

Conservative extension, a purely incremental refinement analogous to conservative theory extensions in logic, is typically used to introduce new behaviour in a modular fashion, like exception handling, robustness features, etc. To define a conservative extension of a given machine, one has to do the following:

- Define the condition for the 'new case' in which the extended machine should execute and the given machine either has no well-defined behaviour or should be prevented from executing. For example, to add error handling to a machine, this condition can be expressed by a shared Boolean-valued error function which is supposed to become true when an emergency has happened and to be reset by the added exception-handling machine.

- Define the new machine with the desired additional behaviour, in the example an exception-handling machine which is executed in case an error has been thrown. The return from the 'new' to the 'old' machine, if there is any, is typically a result of the computation of the new machine.

- Add the new machine and restrict the given machine to the 'old case' by guarding it by the negation of the 'new case' condition, in the example error $\neq$ true.

A real-life example which follows this rather frequent pattern is the refinement of the Java machine by a proven to be correct exception-handling mechanism in [SSB01, Ch. 6] or the incorporation of a bytecode verifier machine into the JVM interpreter in [SSB01, Ch. 17]. As one reviewer pointed out, the use of 'extension' in LOTOS [BoB87] seems to be similar to the conservative extension refinement described here.

\subsection{Procedural Refinement}

Procedural refinement, also called submachine refinement, consists in replacing in a given machine one machine by another (usually more complex) machine. A characteristic example is the refinement of the Prolog ASM which uses an abstract function unify to a machine which calls a submachine implementing a unification procedure [BoR95b] - the example mentioned above of a $(1, n)$-refinement where $n$ can be determined only dynamically since it depends on the size of the to-be-unified terms. In fact such procedural $(1, n)$-refinements with $n>1$ have their typical use in compiler verification when replacing a source code instruction by a chunk of target code; for numerous examples see [BoD96, BoD95, ZiG97, Heb00, GoZ00]. A convenient way to hide the multiplicity of steps of such a refining submachine is to use the concept of ASM submachines which has been defined in [BoS00a] and allows to 'view' $n$ submachine steps as one step of an overall (here the unrefined more abstract) computation. For a specialisation of such submachine refinements to incorporate functional programming techniques see [BoB03].

In general there is a difference between procedural refinement and the above discussed principle of substitutivity. Namely, depending on the granularity of the submachine, in the refined machine one may be able to observe new features which cannot be observed in the abstract machine, although they are related by the refinement definition to the behaviour of the high-level machine.

Procedural Refinement of Control State ASMs. A frequent special case of procedural refinement derives from the graph structure of control state ASMs, a generalisation of Finite State Machines (FSMs) to a class of Abstract State Machines (ASMs) introduced in [Bor99] and extensively used in the AsmBook [BoS03]. They are defined as ASMs all of whose rules have the following form, where $c t l \_s t a t e s$ represent the so-called internal states of FSMs: 


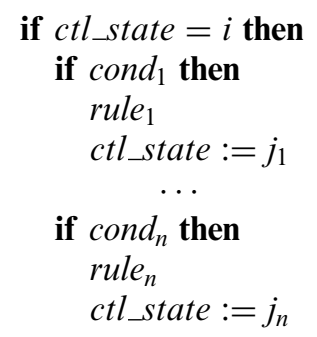

In a given control state $i$, these machines do nothing when no condition $\operatorname{cond}_{k}$ is satisfied; otherwise for every cond $_{k}$ which is satsfied, rule $k$ is executed and the control state changes to $j_{k}$ so that usually the conditions are supposed or guaranteed to be disjoint to avoid conflicting updates which would stop the ASM computation. Control state ASMs represent a normal form for UML activity diagrams (see [BCR00]) from where they inherit the graphical representation of control states by circles or by (possibly named) directed arcs, to visually distinguish the control-passing role of control states from that of the update actions concerning the underlying data structure which are expressed by the ASM rules inscribed into rectangles, separated from the rule guards written into rhombs. Control state ASMs thus offer to use arbitrarily complex parallel (synchronised) data structure manipulations below the main control structure of finite state machines. The overall FSM control structure is reflected by the following notation:

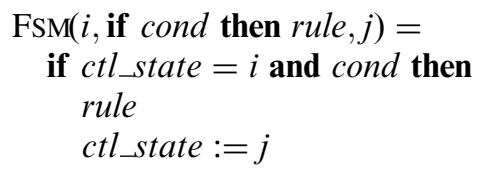

Using this notation the control state ASM rule above becomes the set of rules $\operatorname{FSM}\left(i\right.$, if $\operatorname{cond}_{k}$ then $\left.\operatorname{rule}_{k}, j_{k}\right)$ for $k=1, \ldots, n$.

The procedural refinement of control state ASMs which is suggested by the FSM diagram notation consists in replacing a control state transition - a machine rule at a node with well-defined 'entries' $i$ and 'exits' $j$ - by a new submachine $M$ with the same number of entries and exits, formally replacing $\operatorname{Fsm}(i, r u l e, j)$ by $\operatorname{FsM}(i, M, j)$ (tacitly assuming the renaming of the entry/exit nodes of $M$ to the given ones $i, j$, which is taken care of by the diagram notation).

The reader should be aware that due to the synchronous parallelism of ASMs, in a procedural ASM refinement an action - a part of a parallel step, not limited to a single 'operation' - can be replaced by multiple parallel actions which are viewed as part of a new parallel step. To state it technically, a rule can be refined by finitely many other rules which are executed in parallel. We mention two examples from programming language semantics and from a debugger re-engineering case study. In order to separate the treatment of the semantics of Java thread execution from thread scheduling, in [SSB01, Ch. 7.2] a machine EXECJAVATHREAD is defined using a submachine EXECJAVA. This submachine is refined to the parallel composition of four modules EXECJAVA $I$ dealing with imperative control constructs, EXECJAVA $O$ dealing with object-oriented language features, EXECJAVA describing exception handling, and EXECJAVA ${ }_{T}$ specifying thread-related language constructs. This refinement allowed us to define the semantics instruction-wise so that it can easily be extended to modify or add new language elements. An example from a re-engineering case study appears in [BBG00, 4.3], where the callback for loading modules in a control state debugger model is refined to first bind in parallel each of the shell's breakpoints to the module in question, and only then to call the debugee to continue. It is noteworthy that, analysing this rather abstract high-level rule and the symmetric rule refinement for unloading of modules in the debugger object model, a mismatch was detected between the way loading and unloading of module callbacks was implemented.

Asynchronous Procedural Refinement of Atomic Actions. An example of a procedural refinement which implements an atomic action by multiple actions which are executed in an asynchronous manner is the refinement of the atomic communication between two processes by an asynchronous channel communication in Occam. Channels for a communication to take place require exactly one reader $x$ (positioned to execute an instruction $c ? v$ to read into $\operatorname{val}(v, \operatorname{env}(x))$ the value of channel say bind $(c, e n v(x)))$ and one writer $y$ (positioned to execute an instruction $d ! t$ to write $\operatorname{val}(t, \operatorname{env}(y))$ into the same channel bind $(d, e n v(y)))$. The following instantaneous channel communication rule 


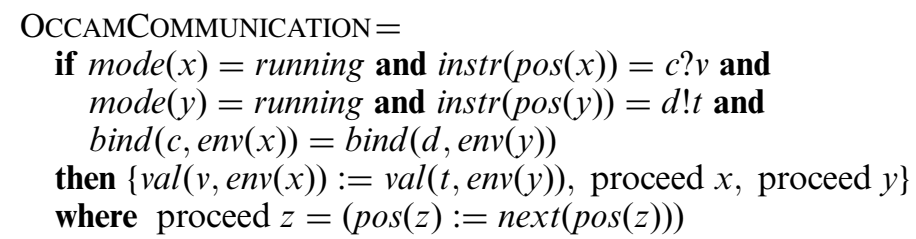

has been refined in [BDR94] as follows by introducing a channel agent which establishes the communication once a reader and a writer have arrived independently recording their identity and variable respectively message.

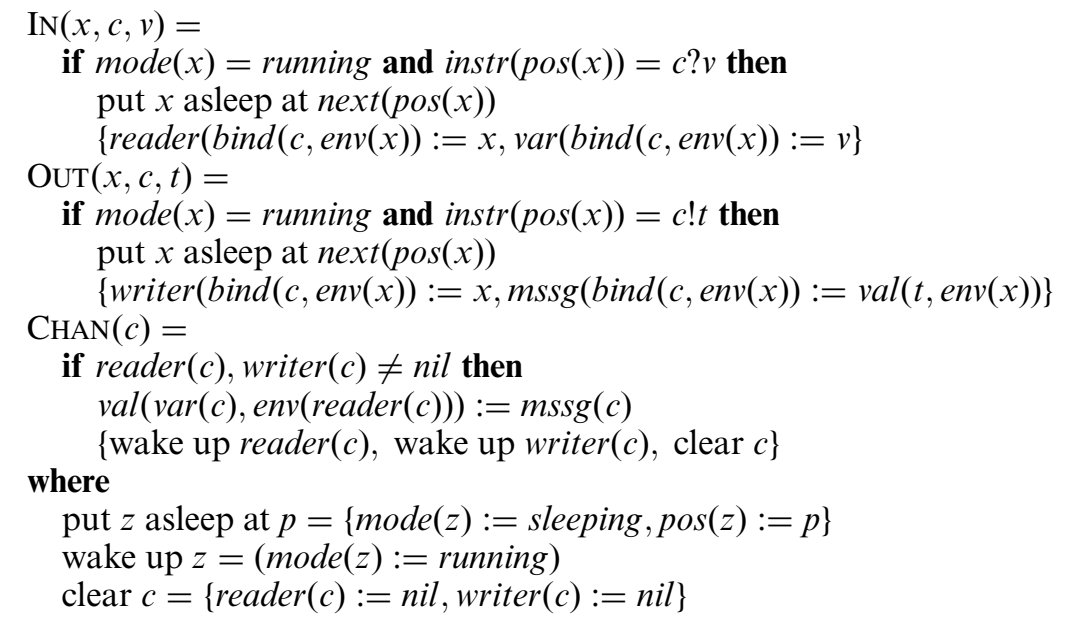

A study of the role of timing constraints for proving the correctness of refinements of asynchronous ASMs with continuous time appears in [CoS00], based upon the ASM models for Lamport's Bakery algorithm in [BGR95] as a case study.

\subsection{Data Refinement}

Many data refinements are given by $(1,1)$-refinements where abstract states and rules are mapped to concrete ones in such a way that the effect of each concrete operation on concrete data types is the same as the effect of the corresponding abstract operation on abstract data types. Pure data refinements are the basis for numerous algebraic and set-theoretic refinement notions [deE98, DeB01], including those used in VDM [FiL98], Z [WoD96] and B [Abr96]. Typically they are used there for corresponding operations with unchanged signature, tailored to provide 'unchanged' properties. For example forward simulation is used to carry over equivalence from pre-states to post-states, backward simulation to carry over equivalence from post-states to pre-states.

A frequently used special type of ASM data refinements which exploits the generalisation of the concept of 'operation' to 'ASM rule' is provided by what is called instantiation where the ASM rules remain unchanged and only the abstract (mostly the external) functions and predicates occurring in them are specified further. Instantiation turned out to be rather useful for the transition in requirements engineering from a use case model with abstract (symbolic) rules to a model which assigns a state transformation meaning to the rule names. Numerous such examples which for reasons of space cannot be exposed here can be found in the AsmBook [BoS03]. We provide here two small examples to illustrate ASM data refinements. In the first example we define an ASM to compute a backtracking scheme which captures the notion of tree generation and traversal in such a way that applying to this machine appropriate data refinements yields well-known logic and functional programming patterns and generative grammars (context-free and attribute grammars). In the second, more theoretical, example we show how classical automata models can be obtained by specialisation of the predicates, functions and updates which constitute Turing-like ASMs.

Tree Traversal. The BACKTRACK machine below dynamically constructs a tree of alternatives and controls its traversal. When its mode is ramify, the submachine RAMIFY creates as many new children nodes to be computation candidates for its currnode as there are computation alternatives, provides them with the necessary environment and switches to selection mode. In mode $=$ select, if at currnode there is no more candidate the 
machine BACKtracks, otherwise the submachine SELECT lets the control move to TRYNEXTCANDIDATE to get executed. The external function alternatives determines the solution space depending upon its parameters and possibly the current state. The dynamic function env records the information every new node needs to carry out the computation determined by the alternative it is associated with. The macro BACK moves currnode one step up in the tree, to parent(currnode), until the root is reached where the computation stops. TRYNEXTCANDIDATE moves currnode one step down in the tree to the next candidate, where next is a possibly dynamic choice function which determines the order for trying out the alternatives. Typically the underlying execution machine EXECUTE will update mode from execute to ramify, in case of a successful execution, or to select if the execution fails. This machine for tree computations is summarised by the following definition:

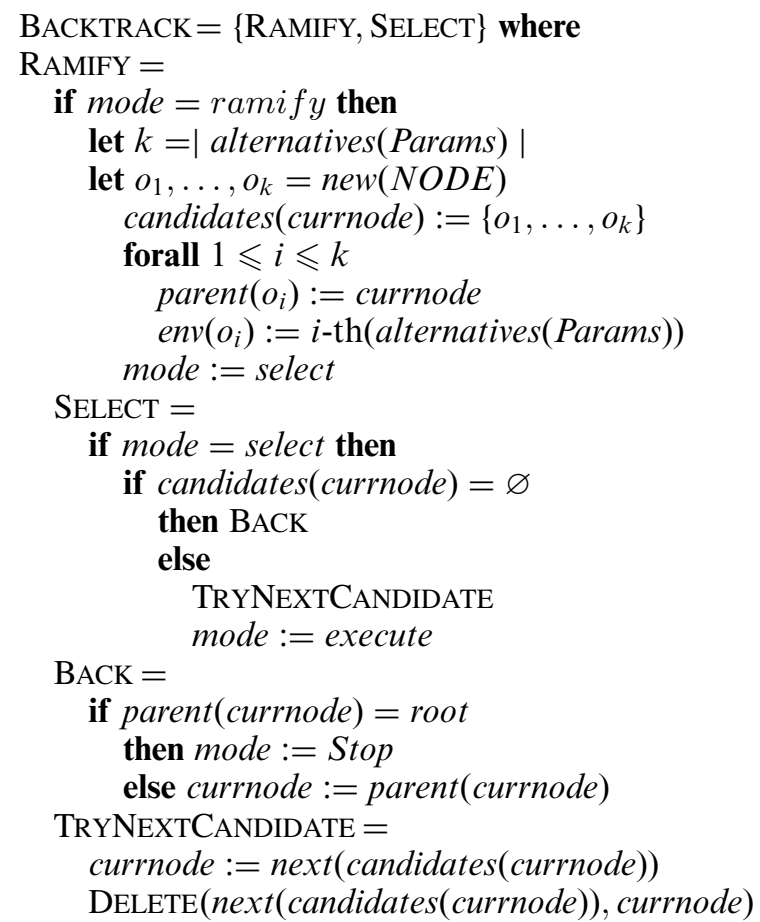

We show now that by data refinements BACKTRACKcan be turned into the backtracking engine for the core of ISO Prolog [BoD90], of IBM's constraint logic programming language CLP(R) [BoS95], of the functional programming language Babel [BLR94], and of context-free and attribute grammars [JoM94].

Data Refinement to Logic Programming Engine. BACKTRACK can be data refined to the backtracking engine for Prolog by instantiating the function alternatives to the function procdef (stm,pgm). This is a Prolog specific function which yields the sequence of clauses in pgm to be tried out in this order to execute the current goal stm; these clauses come together with the needed state information from currnode. We determine next as head function on sequences, reflecting the depth-first left-to-right tree traversal strategy of ISO Prolog. It remains to add the execution engine for Prolog specified as ASM in [BoD90, BoR95a], which switches mode to ramify if the current resolution step succeeds and otherwise switches mode to select.

The backtracking engine for CLP(R) is the same; one only has to extend procdef by an additional parameter for the current set of constraints for the indexing mechanism and to add the CLP(R) engine specified as ASM in [BoS95].

The functional language Babel uses the same function next, whereas the function alternatives is instantiated to fundef (currexp, pgm) yielding the list of defining rules provided in pgm for the outer function of currexp. The Babel execution engine specified as ASM in [BLR94] applies the defining rules in the given order to reduce $c u r r e x p$ to normal form (using narrowing, a combination of unification and reduction).

Data Refinement to Context-Free and Attribute Grammars. To instantiate BACKTRACK for context free grammars $G$ generating leftmost derivations we define the function alternatives(currnode, $G$ ) to yield the sequence of symbols $Y_{1}, \ldots, Y_{k}$ of the conclusion of a $G$-rule whose premise $X$ labels currnode, so that env records the label of 
a node, either a variable $X$ or terminal letter $a$. The definition of alternatives includes a choice between different rules $X \rightarrow w$ in $G$. For leftmost derivations next is defined as for Prolog. As machine in mode = execute one can add the following rule. For nodes labelled by a variable it triggers further tree expansion; for terminal nodes it extracts the yield (concatenating the terminal letter to the word generated so far) and moves the control to the parent node to continue the derivation in mode $=$ select.

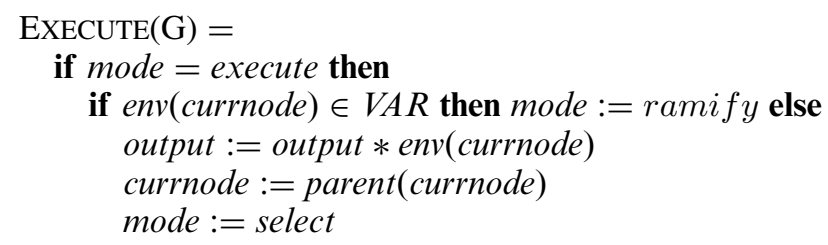

For attribute grammars it suffices to extend the instantiation for context-free grammars as follows. For the synthesis of the attribute $X$. $a$ of a node $X$ from its children's attributes we add to the else-clause of the BACK macro the corresponding update, e.g. $X . a:=f\left(Y_{1} \cdot a_{1}, \ldots, Y_{k} \cdot a_{k}\right)$, where $Y_{i}=e n v\left(o_{i}\right)$ for children nodes $o_{i}$ and $X=e n v($ parent(currnode)). Inheriting an attribute from the parent and siblings can be included in the update of env (e.g. upon node creation), extending it to update also node attributes. The attribute conditions for grammar rules are included in $\operatorname{EXECUTE}(\mathrm{G})$ as an additional guard to yielding output, of the form Cond(currnode.a, parent(currnode).b, siblings(currnode).c).

In a similar way one can formulate an ASM for tree-adjoining grammars, generalising Parikh's analysis of context-free languages by 'pumping' of context-free trees from basis trees (with terminal yield) and recursion trees (with terminal yield except for the root variable), see [JoM94].

Instantiating Turing-Like ASMs. We define here a TURINGLIKEMACHINE and specialise it by instantiation of its predicates, functions and updates to the following examples of classical automata models: 1-way or 2-way finite state machines, timed automata, pushdown automata, Turing machines, Eilenberg's X-machines, Wegner's interactive Turing machines. For further specialisations, including models of distributed computation like Petri nets, we refer the reader to [Bor02a].

Turing-like machines are control state ASMs which in each step, placed in a certain position of their memory, check a Condition concerning the memory in the environment of that position and react by updating mem(env(pos)) and pos. Variations of these machines are due to variations of mem, pos, env, Cond and of the Updates, whereas their rules are all of the following form:

\section{TuringLikeMAChine (mem, pos, env, Cond, Update $)=$ $\operatorname{Fsm}(i$, if $\operatorname{Cond}(\operatorname{mem}(\operatorname{env}($ pos $)))$ then $\operatorname{Update}(\operatorname{mem}(\operatorname{env}($ pos $))$, pos $), j)$}

The specialisation of TURINGLIKEMACHINE to 1-way finite automata in the sense of Moore is obtained by specifying mem as input function in ranging over letters $a$ of an alphabet $A$. Since the monitored function in is 0 -ary there is no pos or env to specify and Cond becomes in $=a$. Since Moore automata emit no output, the Update of mem is empty. So this refinement is a combination of a rather simple data refinement and an equally simple operation refinement.

1-way Mealy automata are obtained by adding to the memory of Moore automata also output, represented by a 0 -ary function out ranging over letters $b$ of an output alphabet $B$. Formally the data refinement yields $m e m=($ in,$o u t)$ and the operation refinement lets Update become the assignment out $:=b$, which changes the output part of mem.

The generalisation of 1-way to 2-way Mealy/Moore automata comes up to specify in not as a 0 -ary but as monadic function (a 'tape') with a dynamic reading head position (where env always takes value pos). As a consequence of this data refinement, Cond reads $i n(p o s)=a$. For the operation refinement an assignment pos $:=$ pos + move is added to Update.

Obviously instead of letting in, out range over letters $a, b$ one may also have words or other value types and also sets or sequences of input or output lines (ports), like in networks of finite automata [BPR84] or stream FSMs [Jan00]. All such examples, obtainable by data and operation refinements similar to those exhibited above, are instances of Mealy/Moore ASMs defined in [Bor99] as TURINGLIKEMACHINEs with arbitrary mem (including in) and any ASM rule as Update, but with the typical FSM Condition $i n=a$. There are numerous examples of such MEALYASMs in the literature, e.g. the components of co-design FSMs (see [Lav00]) where the ASM rules compute arbitrary combinational (external and instantaneous) functions. Another famous example is constituted 
by timed automata [AlD94] where the letter input comes at a real-valued occurrence time which is used in the transitions with clocks recording the time difference of the current input with respect to the previous input:

time $_{\triangle}=$ occurrenceTime(in) - occurrenceTime(previousIn)

The rule Condition is specified as $\operatorname{Timed} \operatorname{In}(a)$, reflecting that transitions may be subject to clock constraints. Typically the constraints are about input to occur within $(<, \leqslant)$ or after $(>, \geqslant)$ a given (constant) time interval, leaving some freedom for timing runs, i.e. choosing sequences of occurrence Time (in) to satisfy the constraints. The memory Update $=$ ClockUpdate is about resetting a clock (namely for all elements of a set Reset) or adding to it the last input time difference. This instantiation of TURINGLIKEMACHINE to timed automata yields the following form of control state ASM rules:

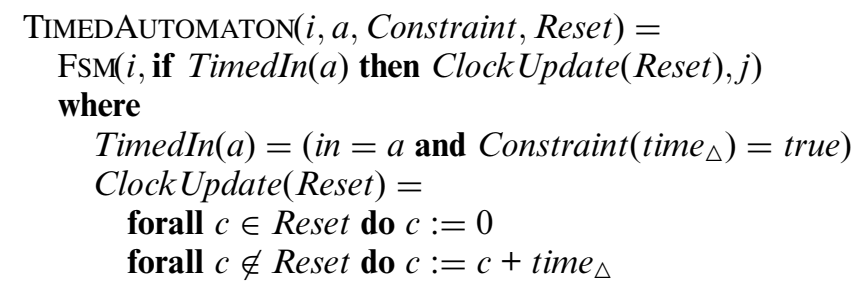

In pushdown automata the Mealy automaton 'reading from input' and 'writing to output' is extended to reading from input and/or a stack and writing on the stack, formally specifying mem = (in, stack) with a function pos $=\operatorname{top}($ stack $)$. Therefore Cond becomes Reading $(a, b)$ from input and/or stack and Update becomes a StackUpdate, resulting in the following form of ASM control state rules. Since PDAs may have control states with no input-reading or no stack-reading, optional items are enclosed in []. We assume the usual meaning of the stack operations push,pop.

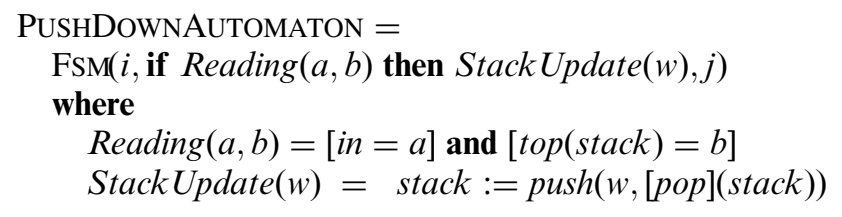

For the original Turing machines TURINGLIKEMACHINE is instantiated by specifying mem as a tape for both reading (input) and writing (output), with an integer position pos: $\mathcal{Z}$ of the reading head on the tape where single letters are retrieved. Thus the reading Condition becomes tape $(p o s)=a$ and Update like in 2-way FSMs concerns both mem and pos, namely tape $($ pos $):=b$ for some $b$ and pos $:=$ pos + move for some move $\in\{1,0,-1\}$.

The extension of the 1-tape Turing machine to a $k$-tape or to an $n$-dimensional TM results from data refining further the 1-tape Turing memory and the related operations and functions. Register machines are a data-refined instance of $k$-tape Turing machines ([Bor89, Ch. AI1]). Eilenberg's $X$-machines [Eil74] (and similarly their streamprocessing version) instead of read/write operations on words stored in a tape provide data processing for arbitrary data, residing in abstract memory, by arbitrarily complex global mem-transforming functions. Therefore they can be instantiated like Mealy ASMs whose rules in addition to yielding output also update mem via global memory functions $f$ (one for each input and control state):

$$
\mathrm{XMACHINE}=\operatorname{FSM}(i, \text { if } \text { in }=a \text { then }\{\text { out }:=b, \text { mem }:=f(\text { mem })\}, j)
$$

If one prefers to write Turing machine programs in the usual tabular form, with one entry $(i, a, j, b, m)$ for every instruction 'in control state $i$ reading input $a$, go to control state $j$, print output $b$ and move the reading head by $m$ ', one obtains the following guard-free Turing machine rule scheme for updating (ctl_state, tape(head), head). The parameters Nxtctl, Write, Move are the three projection functions which define the program table, mapping 'configurations' $(i, a)$ of control state and letter under the reading head to the next control state $j$, the letter $b$ to be written in the reading head position and the move $m$ to be performed by the reading head.

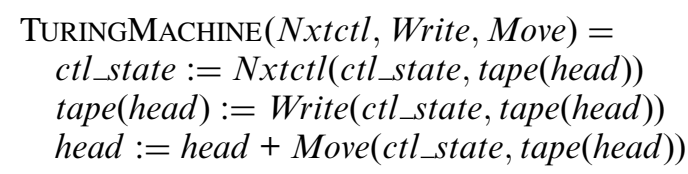


Wegner's interactive Turing machines [Weg97] in each step can receive some input from the environment and yield output to the environment. Thus they simply extend the TURINGMACHINE as follows by an additional input parameter (data refinement) and an output action (operation refinement):

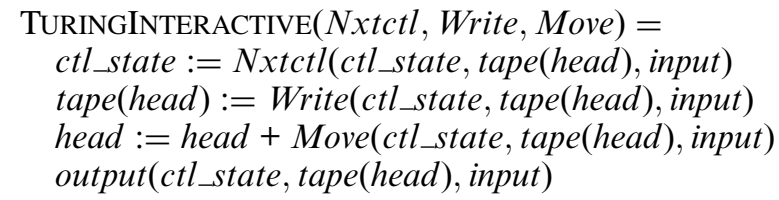

Considering the output as written on an in-out tape comes up to define

output $:=$ concatenate(input, Out(control, tape(head), input))

as the output action using a function Out defined by the program. Viewing the input as a combination of preceding inputs/outputs with the new user input comes up to define input as a derived function input $=$ combine(output, user_input) depending on the current output and user_input. The question of single-stream versus multiple-stream interacting Turing machines (SIM/MIM) is only a question of instantiating input to a stream vector $\left(\right.$ inp $_{1}, \ldots$, inp $\left._{n}\right)$.

\section{Proving Refinement Correctness}

In this section we show how to use ASM refinements for proving system properties. We explain the general scheme and Schellhorn's analysis in [Sch01] for modularising ASM refinement correctness proofs aimed at mechanisable proof support.

The ASM refinement method provides the designer with a powerful method, which has been well known in mathematics for centuries, to $(0)$ show that an implementation $S^{*}$ satisfies a desired property $P^{*}$. Namely instead of directly proving $P^{*}$ for $S^{*}$, one can

1. build an abstract model $S$;

2. prove a possibly abstract form $P$ of the property in question to hold under appropriate assumptions for $S$;

3. show $S$ to be correctly refined by $S^{*}$ and the assumptions to hold in $S^{*}$.

The practice of system design shows that the overall task (0), which for real-life systems is usually too complex to be tackled at a single blow, can be accomplished by splitting it into a series of manageable subtasks (1)-(3), each step reflecting a part of the design. Numerous examples illustrating this use of ASM refinements appear in the AsmBook [BoS03], a large real-life case study which makes wide use of that scheme is the book [SSB01]. A rather complete list of applications of that scheme appears in the ASM research survey in [Bor02b].

As a basis for the machine verification in KIV [ScA97, ScA98] of the proven to be correct hierarchy of ASMs relating Prolog to its compilation to WAM code [BoR95b], Schellhorn has devised in [Sch01] a general scheme for establishing invariants to prove the correctness of an ASM refinement. It is an adaptation of features known from the well-established theory of forward simulations. The idea consists in decomposing the commuting diagram in Fig. 1 into more basic diagrams with end points $s, s^{*}$ which satisfy an invariant $\approx$ implying the to-be-established equivalence $\equiv$. The method is to follow the two runs, for each pair of corresponding states - not both final satisfying $\approx$, looking for a successor pair $s^{\prime}, s^{* \prime}$ (of corresponding states satisfying $\approx$ ). Three cases are possible for such run extensions: only one of the two runs can be extended or both are extendable. These cases give rise to three types of basic diagrams shown in Fig. 2:

- $(m, 0)$-triangles: computation segments where only the abstract run makes progress performing a positive number $m$ of steps to reach an $s^{\prime} \approx s^{*}$;

- $(0, n)$-triangles: computation segments where only the concrete run makes progress performing a positive number $n$ of steps to reach an $s^{* \prime} \approx s$;

- $(m, n)$-trapezoids: representing a computation segment which leads in $m>0$ steps to an $s^{\prime}$ and in $n>0$ steps to an $s^{* \prime}$ such that $s^{\prime} \approx s^{* \prime}$. Any of the three possible subcases $m<n, m>n$ (typical for optimizations) or $m=n$ is allowed here.

To formulate Schellhorn's theorem we first define the Forward Simulation Condition (FSC). It is defined as the following run condition: for every pair $\left(s, s^{*}\right)$ of states, if $s \approx s^{*}$ and not both are final states, then 

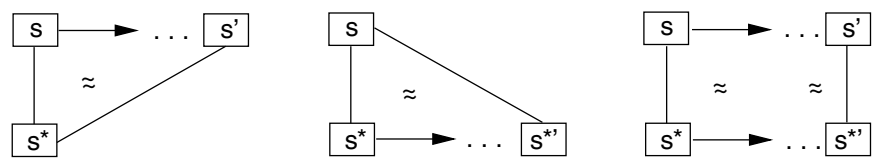

Fig. 2. Components of ASM Refinement Diagrams.

- either the abstract run can be extended by an $(m, 0)$-triangle leading in $m>0$ steps to an $s^{\prime} \approx s^{*}$ satisfying $\left(s^{\prime}, s^{*}\right)<_{m 0}\left(s, s^{*}\right)$ for a well-founded relation $<_{m 0}$ limiting successive applications of $(m, 0)$-triangles;

- or the refined run can be extended by a $(0, n)$-triangle leading in $n>0$ steps to an $s^{*^{\prime}} \approx s$ satisfying the condition $\left(s, s^{* \prime}\right)<_{0 n}\left(s, s^{*}\right)$ for a well-founded relation $<_{0 n}$ limiting successive applications of $(0, n)$-triangles; ${ }^{3}$

- or both runs can be extended by an $(m, n)$-trapezoid leading in $m>0$ abstract steps to an $s^{\prime}$ and in $n>0$ refined steps to an $s^{* \prime}$ such that $s^{\prime} \approx s^{* \prime}$.

Schellhorn's Theorem on Decomposition of ASM Refinement Diagrams in [Sch01] can now be formulated as follows; it has been proved using the KIV verification system. $M^{*}$ is a correct refinement of $M$ with respect to an equivalence notion $\equiv$ and a notion of initial/final states if there is a relation $\approx$ (a coupling invariant) such that

1. The coupling invariant implies the equivalence.

2. Each refined initial state $s^{*}$ is coupled by the invariant to an abstract initial state $s \approx s^{*}$.

3. The forward simulation condition FSC holds.

Also in [Do198] generic PVS theories are described which define refinement relations between ASMs, without allowing arbitrary non-determinism or triangles. The PVS-based approach to mechanical verification of ASMs has been applied in [ZiG97, DGV98] to prove the correctness of back-end rewrite system specifications with ASM-specified source and target languages.

\section{Conclusion: Using ASM Refinements}

In this paper we have explained the ASM refinement method. We have defined the general notion of ASM refinement (Sect. 3) and have shown (Sect. 4) that some of its specialisations integrate other refinement approaches in the literature. In Sect. 5 we have analysed a general correctness proof scheme for ASM refinements, which turned out to be useful for analysing simulations of abstract runs by concrete runs. Summarising one can say that the main usage of ASM refinements is directed towards capturing orthogonalities by modular machines. One way to exhibit orthogonalities is the construction of hierarchical levels for horizontal piecemeal extensions and adaptations of systems (design for change), as experienced for the first time by the numerous extensions of the ISO Prolog ASM model in [Bor90a, Bor90b, Bor92, BoD90] by constraints (for Prolog III in [BoS91]), polymorphism (for Protos-L in [BeB92, BeB96b, BeB96a]), narrowing (for Babel in [BLR94]), object orientation (see [Mul94b, Mul94a]), parallelism (Parlog, Concurrent Prolog etc. in [Ric91, BoR92a, BoR93, Ric92]), and an abstract execution strategy (language Gödel in [BoR94]). Another direction of laying down orthogonalities consists in the use of ASM refinements for the vertical stepwise detailing of models (design for reuse) in a proven to be correct way down to their implementation, as experienced for the first time by the model chains leading from Prolog to WAM code [BoR95b], from Occam to Transputer code [BoD96] and from the serial to the pipelined RISC architecture DLX [BoM97a] which initiated the use of the ASM refinement method for design-driven architecture verification [HuV98, TWF00]. Such ASM refinements support an effective reuse of models and proof techniques, as experienced for the first time reusing the Prolog-to-WAM compilation models and analysis for compiling CLP(R) to IBM's Constraint Logic Arithmetical Machine CLAM [BoS95] and for the compilation of Protos-L to IBM's Protos Abstract Machine [BeB96b, BeB96a].

An important practical effect of using ASM refinements which scales to industrial-size systems is the enhancement of the communication of designs and of system documentations, based upon the report of the design decisions which led to refinement steps and to the accompanying analysis and justification. Outstanding examples

\footnotetext{
3 This well-founded order condition is guaranteed in refinements of event-based B systems by the VARIANT clause, containing an expression for a natural number which has to be shown to decrease by each rule application [AbM98].
} 
which can be mentioned in this context are standardisation efforts which have been supported by ASM modelling and analysis work, namely for the ISO standard of Prolog [BoD90, BoR95a], for the IEEE standard of VHDL'93 [Sta93, BGM94, BGM95] and for the ITU standard of SDL-2000 [ITU00, Gla99, GGP99, EGG00, Pri00]. Last but not least, writing manuals and system maintenance are supported by the accurate, precise, richly indexed and easily searchable documentation coming with refinement reports in electronical form. As an example we refer to the documentation of the Java programming language and its implementation on the Java Virtual Machine provided by the ASM models for Java/JVM and their analysis in [SSB01] which, following a recent evaluation in [HaM01, Section 6.2], '... gives the most comprehensive and consistent formal account of the combination of Java and the JVM, to date'.

\section{Acknowledgements}

We thank E. Boiten (Canterbury), M.-L. Potet (Grenoble), G. Schellhorn (Augsburg) and two anonymous referees for valuable critical comments on the previous versions of this paper.

\section{References}

[Abr96] Abrial, J. -R.: The B-Book. Cambridge University Press, 1996.

[AbM98] Abrialm, J. -R. and Mussat, L.: Introducing dynamic constraints in b. In Bert, D., editor, B'98: Recent Advances in the Development and Use of the B Method, volume 1393 of LNCS, Springer, 1998, pp. 82-128.

[AlD94] Alur, R. and Dillm, D. L.: A theory of timed automata. Theoretical Computer Science, 126:183-235, 1994.

[Bac79] Back, R. J. R.: On correct refinement of programs. Journal of Computer and System Sciences, 23(1):49-68, 1979.

[Bav98] Back, R. J. R. and von Wright, J.: Refinement Calculus: A Systematic Introduction. Springer, 1998.

[BBG00] Barnett, M., Börger, E., Gurevich, Y., Schulte, W. and Veanes, M.: Using Abstract State Machines at Microsoft: A case study. In Y. Gurevich, P. Kutter, M. Odersky and L. Thiele, editors, Abstract State Machines: Theory and Applications, volume 1912 of $L N C S$, Springer 2000, pp. 367-380.

[BeB92] Beierle, C. and Börger, E.: Correctness proof for the WAM with types. In E. Börger, G. Jäger, H. Kleine Büning and M. M. Richter, editors, Computer Science Logic, volume 626 of LNCS, Springer 1992, pp. 15-34.

[BeB96a] Beierle, C. and Börger, E.: Refinement of a typed WAM extension by polymorphic order-sorted types. Formal Aspects of Computing, 8(5):539-564, 1996.

[BeB96b] Beierle, C. and Börger, E.: Specification and correctness proof of a WAM extension with abstract type constraints. Formal Aspects of Computing, 8(4):428-462, 1996.

[BoB87] Bolognesi, T. and Brinksma, E.: Introduction to the ISO specification language LOTOS. Computer Networks and ISDN Systems, 14(1):25-59, 1987.

[Bor89] Börger, E.: Computability, Complexity, Logic (English translation of Berechenbarkeit, Komplexität, Logik), volume 128 of Studies in Logic and the Foundations of Mathematics. North-Holland, 1989.

[Bor90a] Börger, E.: A logical operational semantics for full Prolog. Part I: Selection core and control. In Börger, E., Kleine Büning, H., Richter, M. M. and Schönfeld, W., editors, CSL'89. 3rd Workshop on Computer Science Logic, volume 440 of LNCS, Springer, 1990, pp. 36-64.

[Bor90b] Börger, E.: A logical operational semantics of full Prolog. Part II: Built-in predicates for database manipulation. In B. Rovan, editor, Mathematical Foundations of Computer Science, volume 452 of LNCS, Springer, 1990, pp. 1-14.

[Bor92] Börger, E.: A logical operational semantics for full Prolog. Part III: Built-in predicates for files, terms, arithmetic and inputoutput. In Y. Moschovakis, editor, Logic From Computer Science, volume 21 of Berkeley Mathematical Sciences Research Institute Publications, Springer, 1992, pp. 17-50.

[Bor94] Börger, E.: Logic programming: The evolving algebra approach. In B. Pehrson and I. Simon, editors, IFIP 13th World Computer Congress, volume I: Technology/Foundations, Elsevier, Amsterdam, 1994, pp. 391-395.

[Bor99] Börger, E.: High level system design and analysis using abstract state machines. In D. Hutter, W. Stephan, P. Traverso and M. Ullmann, editors, Current Trends in Applied Formal Methods (FM-Trends 98), volume 1641 of LNCS, Springer, 1999, pp. 1-43.

[Bor02a] Börger, E.: Computation and specification models: a comparative study. In P. D. Mosses, editor, Proceedings of the Fourth International Workshop on Action Semantics, volume NS-02-8 of BRICS Series, Department of Computer Science at University of Aarhus, December 2002, pp. 107-130.

[Bor02b] Börger, E.: The origins and the development of the ASM method for high level system design and analysis. Journal of Universal Computer Science, 8(1):2-74, 2002.

[Bor03] Börger, E.: Abstract State Machines: a unifying view of models of computation and of system design frameworks. Annals of Pure and Applied Logic, 2003.

[BoB03] Börger, E. and Bolognesi, T.: Remarks on turbo ASMs for computing functional equations and recursion schemes. In E. Börger, A. Gargantini and E. Riccobene, editors, Abstract State Machines 2003, volume 2589 of LNCS. Springer, 2003.

[BCR00] Börger, E., Cavarra, A. and Riccobene, E.: An ASM semantics for UML activity diagrams. In T. Rus, editor, Algebraic Methodology and Software Technology, 8th International Conference, AMAST 2000, Iowa City, Iowa, USA, May 20-27, 2000, volume 1816 of LNCS, Springer 2000, pp. 293-308.

[BoD90] Börger, E. and Dässler, K.: Prolog: DIN papers for discussion. ISO/IEC JTCI SC22 WG17 Prolog Standardisation Document 58, National Physical Laboratory, Middlesex, UK, 1990. 
[BoD95] Börger, E. and Del Castillo, G.: A formal method for provably correct composition of a real-life processor out of basic components (APE100 Reverse Engineering Study). In B. Werner, editor, Proceedings of the First IEEE International Conference on Engineering of Complex Computer Systems (ICECCS'95), November 1995, pp. 145-148.

[BoD96] Börger, E. and Durdanović, I.: Correctness of compiling Occam to Transputer code. Computer Journal, 39(1):52-92, 1996.

[BDR94] Börger, E., Durdanović, I. and Rosenzweig, D.: Occam: specification and compiler correctness. Part I: Simple mathematical interpreters. In U. Montanari and E. R. Olderog, editors, Proceedings of PROCOMET'94 (IFIP Working Conference on Programming Concepts, Methods and Calculi), North-Holland, 1994, pp. 489-508.

[BGM94] Börger, E., Glässer, U. and Müller, W.: The semantics of behavioral VHDL'93 descriptions. In EURO-DAC'94. European Design Automation Conference with EURO-VHDL'94, Los Alamitos, CA, 1994. IEEE Computer Society Press, pp. 500-505.

[BGM95] Börger, E., Glässer, U. and Müller, W.: Formal definition of an abstract VHDL'93 simulator by ea-machines. In C. Delgado Kloos and P. T. Breuer, editors, Formal Semantics for VHDL, Kluwer, 1995, pp. 107-139

[BGR95] Börger, E., Gurevich, Y. and Rosenzweig, D.: The bakery algorithm: yet another specification and verification. In E. Börger, editor, Specification and Validation Methods, Oxford University Press, 1995, pp. 231-243

[BLR94] Börger, E., López-Fraguas, F. J. and Rodríguez-Artalejo, M.: A model for mathematical analysis of functional logic programs and their implementations. In B. Pehrson and I. Simon, editors, IFIP 13th World Computer Congress, volume I: Technology/Foundations, Elsevier, Amsterdam, 1994, pp. 410-415.

[BoM97a] Börger, E. and Mazzanti, S.: A practical method for rigorously controllable hardware design. In J. P. Bowen, M. B. Hinchey and D. Till, editors, ZUM'97: The Z Formal Specification Notation, volume 1212 of LNCS, Springer, 1997, pp. $151-187$.

[BoM97b] Börger, E. and Mearelli, L.: Integrating ASMs into the software development life cycle. Journal of Universal Computer Science, 3(5):603-665, 1997.

[BoR92a] Börger, E. and Riccobene, E.: A mathematical model of concurrent Prolog. Research Report CSTR-92-15, Department of Computer Science, University of Bristol, UK, 1992.

[BoR93] Börger, E. and Riccobene, E.: A formal specification of Parlog. In M. Droste and Y. Gurevich, editors, Semantics of Programming Languages and Model Theory, Gordon \& Breach, 1993, pp. 1-42.

[BoR94] Börger, E. and Riccobene, E.: Logic + control revisited: an abstract interpreter for Gödel programs. In G. Levi, editor, Advances in Logic Programming Theory, Oxford University Press, 1994, pp. 231-154.

[BoR91] Börger, E. and Rosenzweig, D.: From Prolog algebras towards WAM: a mathematical study of implementation. In E. Börger, H. Kleine Büning, M. M. Richter and W. Schönfeld, editors, CSL'90, 4th Workshop on Computer Science Logic, volume 533 of $L N C S$, Springer 1991, pp. 31-66.

[BoR92b] Börger, E. and Rosenzweig, D.: WAM algebras: a mathematical study of implementation, Part 2. In A. Voronkov, editor, Logic Programming, volume 592 of Lecture Notes in Artificial Intelligence, Springer, 1992, pp. 35-54.

[BoR95a] Börger, E. and Rosenzweig, D.: A mathematical definition of full Prolog. Science of Computer Programming, 24:249-286, 1995.

[BoR95b] Börger, E. and Rosenzweig, D.: The WAM: definition and compiler correctness. In C. Beierle and L. Plümer, editors, Logic Programming: Formal Methods and Practical Applications, Studies in Computer Science and Artificial Intelligence, chapter 2, North-Holland, 1995 , pp. 20-90.

[BoS95] Börger, E. and Salamone, R.: CLAM specification for provably correct compilation of CLP $(\mathcal{R})$ programs. In E. Börger, editor, Specification and Validation Methods, Oxford University Press, 1995, pp. 97-130.

[BoS00a] Börger, E. and Schmid, J.: Composition and submachine concepts for sequential ASMs. In P. Clote and H. Schwichtenberg, editors, Computer Science Logic (Proceedings of CSL 2000), volume 1862 of LNCS, Springer, 2000, pp. 41-60.

[BoS91] Börger, E. and Schmitt, P.: A formal operational semantics for languages of type Prolog III. In E. Börger, H. Kleine Büning, M. M. Richter and W. Schönfeld, editors, CSL'90, 4th Workshop on Computer Science Logic, volume 533 of LNCS, Springer, 1991, pp. 67-79.

[BoS98] Börger, E. and Schulte, W.: Programmer friendly modular definition of the semantics of Java. In J. Alves-Foss, editor, Formal Syntax and Semantics of Java, volume 1523 of LNCS. Springer, 1998.

[BoS00b] Börger, E. and Schulte, W.: A practical method for specification and analysis of exception handling: a Java/JVM case study. IEEE Transactions on Software Engineering, 26(10):872-887, October 2000.

[BoS03] Börger, E. and Stärk, R.: Abstract State Machines: A Method for High-Level System Design and Analysis. Springer, 2003.

[BPR84] Brüggemann, A., Priese, L., Rödding, D. and Schätz, R.: Modular decomposition of automata. In E. Börger, G. Hasenjäger and D. Rödding, editors, Logic and Machines: Decision Problems and Complexity, volume 171 LNCS, Springer, 1984, pp. 198-236.

[CoS00] Cohen, J. and Slissenko, A.: On verification of refinements of asynchronous timed distributed algorithms. In Y. Gurevich, P. Kutter, M. Odersky and L. Thiele, editors, Abstract State Machines: Theory and Applications, volume 1912 of LNCS, Springer, 2000, pp. $34-49$.

[DDH72] Dahl, O., Dijkstra, E. W. and Hoare, C.: Structured Programming. Academic Press, 1972.

[deE98] de Roever, W. P. and Engelhardt, K.: Data Refinement: Model-Oriented Proof Methods and their Comparison. Cambridge University Press, 1998.

[DeB01] Derrick, J. and Boiten, E.: Refinement in Z and Object-Z. Springer, 2001.

[Dij72] Dijkstra, E.: Notes on structured programming. In Structured Programming, Academic Press, 1972, pp. 1-82.

[Do198] Dold, A.: A formal representation of Abstract State Machines using PVS. Verifix Technical Report Ulm/6.2, Universität Ulm, July 1998 .

[DGV98] Dold, A., Gaul, T., Vialard, V. and Zimmermann, W.: ASM-based mechanised verification of compiler back-ends. In U. Glässer and P. Schmitt, editors, Proceedings of the Fifth International Workshop on Abstract State Machines, Magdeburg University, 1998, pp. 50-67.

[Eil74] Eilenberg, S.: Automata, Machines and Languages Vol. A. Academic Press, 1974.

[EGG00] Eschbach, R., Glässer, U., Gotzhein, R. and Prinz, A.: On the formal semantics of SDL-2000: a compilation approach based on an abstract SDL machine. In Y. Gurevich, P. Kutter, M. Odersky and L. Thiele, editors, Abstract State Machines: Theory and Applications, volume 1912 of LNCS, Springer, 2000, pp. 242-265.

[FiL98] Fitzgerald, J. and Larsen, P. G.: Modelling Systems. Practical Tool and Techniques in Software Development. Cambridge University Press, 1998. 
[Gla99] Glässer, U.: Analysis and Validation of Formal Requirement Specifications in Model-Based Engineering of Concurrent Systems. Habilitationsschrift, University of Paderborn, Germany, 1999.

[GGP99] Glässer, U., Gotzhein, R. and Prinz, A.: Towards a new formal SDL semantics based on Abstract State Machines. In G. v. Bochmann, R. Dssouli and Y. Lahav, editors, SDL'99: The Next Millennium, Proceedings of the 9th SDL Forum, Elsevier Science, 1999, pp. 171-190.

[GoZ00] Goos, G. and Zimmermann, W.: Verifiying compilers and ASMs. In Y. Gurevich, P. Kutter, M. Odersky and L. Thiele, editors, Abstract State Machines: Theory and Applications, volume 1912 of LNCS, Springer, 2000, pp. 177-202.

[Gur85] Gurevich, Y.: A new thesis. Abstracts, American Mathematical Society, page 317, August 1985.

[Gur95] Gurevich, Y.: Evolving algebras 1993: Lipari Guide. In E. Börger, editor, Specification and Validation Methods, Oxford University Press, 1995, pp. 9-36.

[HaM01] Hartel, P. and Moreau, L.: Formalising the safety of Java, the Java Virtual Machine and Java Card. ACM Computing Surveys, 33(4):517-558, 2001.

[Heb00] Heberle, A.: Korrekte Transformationsphase: der Kern korrekter Übersetzer. PhD thesis, Universität Karlsruhe, 2000.

[HuV98] Huggins, J. and Van Campenhout, D.: Specification and verification of pipelining in the ARM2 RISC microprocessor. ACM Transactions on Design Automation of Electronic Systems, 3(4):563-580, October 1998.

[ITU00] ITU-T. SDL formal semantics definition. ITU-T Recommendation Z.100 Annex F, International Telecommunication Union, November 2000

[Jan00] Janneck, J. W.: Syntax and Semantics of Graphs. PhD thesis, ETH Zürich, 2000.

[JoM94] Johnson, D. E. and Moss, L. S.: Grammar formalisms viewed as evolving algebras. Linguistics and Philosophy, 17:537-560, 1994.

[Mor87] Morris, J. M.: A theoretical basis for stepwise refinement. Science of Computer Programming, 9(3), 1987.

[Lav00] Lavagno, L., Sangiovanni-Vincentelli, A. and Sentovitch, E. M.: Models of computation for system design. In E. Börger, editor, Architecture Design and Validation Methods, Springer, 2000, pp. 243-295.

[Mor94] Morgan, C.: Programming from Specifications. Prentice-Hall, 1990, 1994.

[Mul94a] Müller, B.: Eine objektorientierte Prolog-Erweiterung zur Entwicklung wissensbasierter Systeme. PhD thesis, University of Oldenburg, Germany, 1994.

[Mu194b] Müller, B.: A semantics for hybrid object-oriented Prolog systems. In B. Pehrson and I. Simon, editors, IFIP 13th World Computer Congress, volume I: Technology/Foundations. Elsevier, Amsterdam, 1994.

[Pri00] Prinz, A.: Formal Semantics for SDL. Definition and Implementation. Habilitationsschrift, Humboldt University, Berlin, 2000.

[Ric92] Riccobene, E.: A formal computational model for PANDORA. Technical Report CSTR-92-16 and ACRC-92-15, University of Bristol, Department of Computer Science, 1992.

[Ric91] Riccobene, E.: Modelli Matematici per Linguaggi Logici. PhD thesis, University of Catania, Academic year 1991/92.

[Sch01] Schellhorn, G.: Verification of ASM refinements using generalised forward simulation. Journal of Universal Computer Science, 7(11):952-979, 2001.

[ScA97] Schellhorn, G. and Ahrendt, W.: Reasoning about Abstract State Machines: the WAM case study. Journal of Universal Computer Science, 3(4):377-413, 1997.

[ScA98] Schellhorn, G. and Ahrendt, W.: The WAM case study: verifying compiler correctness for Prolog with KIV. In W. Bibel and P. Schmitt, editors, Automated Deduction: A Basis for Applications. Kluwer, 1998.

[Sta93] Standardisation, I.: IEEE standard VHDL language reference manual. Technical Report Std 1076-1993 IEEE. 1993.

[SSB01] Stärk, R. F., Schmid, J. and Börger, E.: Java and the Java Virtual Machine: Definition, Verification, Validation. Springer, 2001.

[TWF00] Teich, J., Weper, R., Fischer, D. and Trinkert, S.: A joint architecture/compiler design environment for asips. In Proceedings of International Conference on Compilers, Architectures and Synthesis for Embedded Systems (CASES2000), ACM Press, November 2000, pp. 26-33.

[Weg97] Wegner, P.: Why interaction is more powerful than algorithms. Communications of the ACM, 40:80-91, 1997.

[Wir71] Wirth, N.: Program development by stepwise refinement. Communications of the ACM, 14(4), 1971 .

[WoD96] Woodcock, J. C. P. and Davies, J.: Using Z: Specification, Refinement, and Proof. Prentice-Hall, 1996.

[ZiG97] Zimmerman, W. and Gaul, T.: On the construction of correct compiler back-ends: An ASM approach. Journal of Universal Computer Science, 3(5):504-567, 1997.

[ZiD03] Zimmermann, W. and Dold, A.: A framework for modelling the semantics of expression evaluation with Abstract State Machines. In E. Börger, A. Gargantini and E. Riccobene, editors, Abstract State Machines 2003: Advances in Theory and Applications, volume 2589 of LNCS. Springer, 2003.

Received October 2002

Accepted March 2003 